\title{
Melatonin-mediated Calcineurin Inactivation Attenuates Prion Protein-induced Nf-Kb- Driven Pro- Inflammation Response
}

\author{
Jeong-Min Hong \\ Jeonbuk National University \\ Ji-Hong Moon \\ Jeonbuk National University \\ Jae-Won Seol \\ Jeonbuk National University \\ Sang-Youel Park ( $\square$ sypark@chonbuk.ac.kr)
}

Jeonbuk National University

\section{Research Article}

Keywords: Prion, Calcineurin, Melatonin, Autophagy, Neuroinflammation

Posted Date: November 30th, 2021

DOI: https://doi.org/10.21203/rs.3.rs-1112914/v1

License: (c) (i) This work is licensed under a Creative Commons Attribution 4.0 International License.

Read Full License 


\section{Abstract}

Background: Prion diseases are a group of prevalent and rapidly progressive neurodegenerative disorders that lead to chronic inflammation and neuronal cell death. Calcineurin and autophagy mediate prioninduced neurodegeneration, suggesting that inhibition of calcineurin and autophagy could be a target for therapy. Melatonin has been reported to exert neuroprotective effects against calcium-dependent neuronal cell death.

Methods: Real-time quantitative PCR was used to detect mRNA levels of proinflammatory cytokines. Western blot was used to analysis p-nfkb, p-bcl10, calcineurin, prpc and autophagy flux pathway. Immunocytochemistry was used to analysis $\mathrm{p}$-nfkb and calcineurin. $\mathrm{Ca}^{2+}$ levels were measured by fluo-4 using confocal microscope. Calcineurin activity was used to detect with calcineurin cellular activity assay kit. Transmission electron microscopy (TEM) was used to detect autophagy flux.

Results: In the present study, we investigated whether melatonin attenuates prion peptide-mediated neuroinflammation and reduces calcineurin. We found that melatonin treatment inhibits prion proteininduced apoptosis. Melatonin inhibited calcium up-regulation and protected the cells against prion peptide-induced neuron cell death by calcineurin inactivation. Furthermore, melatonin increased p62 protein levels and decrease LC3-II protein levels indicating autophagic flux inhibition and melatonin inhibited prion protein-induced neurotoxicity through autophagy flux inhibition.

Conclusions: Taken together, our results illuminate that melatonin attenuated prion protein-induced neurinflammation through calcineurin inactivation and autophagic flux reduction, and also suggest that melatonin may provide effective strategy for therapy against neurodegenerative diseases, including prion diseases.

\section{Background}

Prion diseases are a group of fatal and unusual neurodegenerative disorders of the nervous system caused by the presence of a misfolded of the cellular prion protein; these diseases affect humans and animals including Creutzfeldt-Jakob disease in human and scrapie in sheep [1-3]. They induced inflammatory enzyme COX-2 and pro-inflammatory cytokines such as TNF-a $[4,5]$. Neurotoxic prion protein (PrP) fragment 106-126 (PrP106-126) is commonly used as a model for the investigation of PrPSc neurotoxicity, as it possesses similar physicochemical and pathological properties to PrPSc. It forms amyloid fibrils with high $\beta$-sheet content, shows partial proteinase $\mathrm{K}$ resistance, and is neurotoxic in vitro [6].

Because PrP106-126 has similar physiochemical and pathological properties to PrPSc, the PrP106-126 is often used as toxicity model of PrPSc in prion disease studies. PrP106-126 with a high $\beta$-sheet enriched structure is resistant to protease $\mathrm{K}$ and neurotoxic $[7,8]$. 
Melatonin (N-acetyl-5-methoxytryptamine) is a key endogenous indole amine secreted and released by pineal gland of mammals, including humans, melatonin plays a critical role in the regulation of circadian rhythms and numerous other functions [9]. Melatonin is amphiphilic in nature and acts as a potent free radical scavenger and possesses antioxidant, anti-inflammatory and anti-apoptotic properties [10, 11]. Melatonin has been shown to inhibit microglial activation and reduce proinflammatory cytokine levels in many experimental models, including Alzheimer's disease (AD) [12]. However, the effect of melatonin on neuroinflammation from prion is unknown.

The serine/threonine phosphatase calcineurin plays an important role in many cellular process [13]. Overactivation of calcineurin in neurotoxicity effects induced by prion peptide and amyloidbeta [14]. Calcineurin occurs cytokines and proinflammatory factors in the neurons throughout brain [15, 16]. Calcineurin mediates activation and translocation of NFKB not through direct dephosphorylation of the transcription factor but through activation of a kinase that causes the removal of inhibitory subunits [17].

\section{Autophagy is a cellular process of regulated self-eating through the degradation of cellular}

components. Autophagy is an evolutionarily conserved intracellular degradation process of cytosolic constituents through formation of autophagosomes followed by their fusion with lysosomes, which results in autolysosome formation $[18,19]$. Autophagy plays an important role in protein misfolding diseases and inflammatory diseases [20]. However, the role of autophagy in melatonin-mediated neuroprotective effects is not fully understood.

Our previous study showed that melatonin inhibits prion peptide-induced neuronal apoptosis by inducing autophagy activation [21]. However, the effect of melatonin on prion protein-induced neuroinflammation via calcineurin and autophagy has yet to be reported. In this study, we observed the neuroprotective effects of melatonin against PrP (106-126)-mediated neuroinflammation, calcineurin and autophagy activation.

\section{Methods}

\section{Cell culture}

SK-N-SH cell lines were obtained from the ATCC (Rockville, MD, USA) and cell culture method has been previously described [22]. The mouse neuronal cell lines ZW 13-2 and Zpl 3-4, which were established from the hippocampus of ICR $\left(\mathrm{Prnp}^{+/+}\right)$and Zürich I Prnp ${ }^{-/-}$mice, respectively, were kindly provided by Professor Yong-Sun Kim (Hallym University, Chuncheon, Kangwon-do, South Korea). The cells were grown in DMEM containing $10 \%$ FBS and gentamycin $(0.1 \mathrm{mg} / \mathrm{ml})$ in a humidified incubator maintained at $37^{\circ} \mathrm{C}$ with $5 \% \mathrm{CO}_{2}$.

\section{PrP (106-126) treatment}


Synthetic PrP (106-126) was synthesized as previously described [23]. Sequences of PrP (106-126) used in this study were Lys-Thr-Asn-Met-Lys-His-Met-Ala-Gly-Ala-Ala-Ala-Ala-Gly-Ala-Val-Val-Gly-Gly-Leu-Gly. PrP (106-126) were synthesized by Peptron (Seoul, Korea).

\section{Measurement of $\left[\mathrm{Ca}^{2+}\right] i$}

Measurement of $\mathrm{Ca}^{2+}$ contents was implemented as previously reported [24]. Briefly, cells were plated on collagen-coated confocal dish. The SK-N-SH cells were loaded with $5 \mu$ M Fluo-4 AM (Invitrogen) for 40 min at $37^{\circ} \mathrm{C}$. The image was processed using a confocal microscope (Zeiss). Fluorescence intensity was measured using Image J program $(\mathrm{NIH})$.

\section{Calcineurin activity assay}

SK-N-SH cells were used to determine calcineurin phosphatase activity. Calcineurin activity was measured with calcineurin cellular activity assay kit (\#BML-AK816-0001; Enzo Life Sciences, Inc., Farmingdale, NY, USA) and assay was performed following manufacturer's instructions. Calcineurin activity was measured as previously reported [25].

\section{Immunocytochemistry}

Immunocytochemical analyses were performed on neuroblastoma cells with p-nfkb (MAB3026; Milipore, Burlington, MA, USA) and anti-calcineurin (ab137335; Abcam). Cells were cultured on glass slides (Nalge Nunc International, Naperville, IL, USA), then washed in sterilized TBST for $10 \mathrm{~min}$, blocked for $15 \mathrm{~min}$ with $5 \% \mathrm{FBS}$ in TBST, and incubated overnight at $4{ }^{\circ} \mathrm{C}$ with the primary antibodies diluted with $5 \% \mathrm{FBS}$ in TBST. Alexa Fluor 488-labeled donkey anti-rabbit IgG antibody, diluted 1:1000 (A21206; Molecular Probes, Eugene, OR, USA), was used to visualize expression using fluorescence microscopy.

\section{Quantitative real-time PCR}

Total RNA was extracted from SK cells using Easy-spin Total RNA Extraction Kit (iNtRON Biotechnology, Seoul, Korea). cDNA synthesis was carried out using TaKaRa Prime Script 1st Strand cDNA synthesis kit (TaKaRa Bio, Tokyo, Japan). For quantitative real-time PCR, $1 \mu \mathrm{l}$ of gene-specific primers and SYBR Green Supermix (Bio-Rad Laboratories, Hercules, CA) were used in $20 \mu \mathrm{l}$ of reaction volume. The primers were as follows: TNF-a, forward = 5'TCT CCT TCC TGA TCG TGG C' and reverse = 5' GGT TCA GCC ACT GGA GCT 3'; COX-2, forward = 5' AAA TTC GGT ACA TCC TCG AC 3' and reverse = 5' CAG GAA CTG GAT CAG GAC TT 3'; glyceraldehyde-3-phosphate dehydrogenase (as an internal control): forward = 5' TGC CTC CTG CAC CAA CT 3 ' and reverse $=5$ 'CGC CTG CTT CAC CTT C 3 '. All quantitative PCR reactions were performed on a CFX96 real-time PCR detection system (Bio-Rad).

\section{Western blot analysis}

Western blot analysis was measured as described previously [26]. The antibodies used for immunoblotting were anti-calcineurin (ab137335; Abcam), p-nf kb p65(Ser536) (\#3033; Cell Signaling 
Technology, Danvers, MA, USA), p-bcl10, anti-P62 (\#5114; Cell Signaling Technology), LC3 (Novus Biologicals, Littleton, CO, USA), and anti- $\beta$-actin (A5441; Sigma-Aldrich, St. Louis, MO, USA).

\section{Transmission electron microscopy (TEM)}

TEM was performed as described previously [27]. Briefly, SK-N-SH cells were fixed with $2 \%$ paraformaldehyde and $2 \%$ glutaraldehyde in $0.05 \mathrm{M}$ sodium cacodylate, $\mathrm{pH}$ 7.2. Cells were post-fixed with $1 \%$ osmium tetroxide for further fixation. Specimens were dehydrated in graded ethanol and changed to propylene oxide, and then embedded in Epoxy resin. Images were recorded on a Hitachi H7650 electron microscope (Hitachi, Ltd., Tokyo, Japan; magnification, x10,000) installed at the Center for University-Wide Research Facilities (CURF) at Jeonbuk National University.

\section{Statistical analysis}

All statistical analysis was carried out using GraphPad Prism software (version 5.03; GraphPad Software, Inc., La Jolla, CA, USA). All experiments were expressed as mean \pm standard error. And all experiments were compared using the one-way ANOVA followed by the Tukey test. Results were considered significant at ${ }^{*} p<0.05,{ }^{\star *} p<0.01$ or ${ }^{\star \star \star} p<0.001$, as appropriate.

\section{Results}

\section{Melatonin is protective against prion peptide-mediated inflammatory responses.}

We investigated the role of melatonin against prion peptide-mediated proinflammatory response in SK-NSH cells. Real-time quantitative PCR analysis was then used to measure the mRNA levels of proinflammatory cytokines, TNF-a, and COX-2. We found that PrP (106-126) treatment led to the induction of TNF- $\mathrm{a}$ and COX-2 mRNA and that this induction was decreased by melatonin treatment (Figures $1 \mathrm{~A}$ and $\mathrm{B}$ ). Further, treatment with melatonin reduced the levels of $p$-NF-KB (Figure $2 \mathrm{C}$ ). In agreement with these data, immunocytochemistry experiments showed that $p$-NF-kB protein levels were markedly lower in PrP (106-126) treatment upon melatonin compared with PrP (106-126) treatment (Figure 1D). We confirmed the effects of melatonin on prion peptide-induced $p$-NF-KB in neuronal cells. Prion peptideinduced $p$-NF-KB was decreased by melatonin, as shown by western blot analysis (Figure $2 \mathrm{~A}$ and $2 \mathrm{C}$ ). Prion peptide-induced $p$-bcl10 was decreased by melatonin, as shown by western blot analysis (Figure 2B and 2D).

\section{Melatonin inhibits PrP (106-126)-induced neuroinflammation by calcineurin inactivation}

To determine the effect of melatonin on prion peptide expression in neuronal cells, we investigated the role of prion peptide in altering $\mathrm{Ca}^{2+}$ levels in neuronal cells. $\mathrm{Ca}^{2+}$ content was directly evaluated by prion peptide, and melatonin reduced PrP-induced $\mathrm{Ca}^{2+}$ (Figures $3 \mathrm{~A}$ and $\mathrm{B}$ ). Fluorescence imaging also indicated that baicalein inhibited calcium increase (Figure $3 \mathrm{C}$ ). Based on the known connection between ER stress and perturbation of calcium homeostasis, we next explored the relative ER calcium content in 
SK-N-SH cells. We monitored the passive release of calcium from the ER by inhibiting the SERCA pump with thapsigargin by using the calcium dye Fluo-4. PrP (106-126) treatment diminished ER calcium release after thapsigargin treatment than PrP (106-126) treatment upon melatonin (Figures 3D and E). Fluorescence imaging also indicated that baicalein inhibited calcium increase (Figure 3F). The results showed that prion protein elevated calcineurin activity and melatonin reduced prion peptide-induced calcineurin activity (Figure 4A). In agreement with these data, immunocytochemistry experiments showed that calcineurin protein levels were markedly lower in $\operatorname{PrP}$ (106-126) treatment upon melatonin compared with $\operatorname{PrP}$ (106-126) treatment (Figure 4B). To determine calcineurin activation, $p$-bcl10 were subjected to western blot. The data showed that prion peptide treatment dose-dependently reduced $p$-bcl10 (Figure 4C). Prion peptide-induced $p$-bcl10 was decreased by melatonin, as shown by western blot analysis (Figure 4D).

\section{Melatonin treatment inhibits PrP (106-126)-induced neuroinflammation via autophagy}

We investigated whether melatonin affects prion peptide-induced autophagy. We detected a dosedependent decrease in the late autophagosome markers LC3-II and SQSTM1/p62 in the PrP-treated group compared with the control group in human neuroblastoma cells (Figure 5A and 5B). We found that melatonin increased SQSTM1/p62 and LC3-II (Figure 5C and 5D). We also conducted TEM to establish the effect of lysosomal inhibition of autophagy by melatonin. As shown in Figure 5E, multiple vesicles including double-membraned autophagosomes (arrows) were reduced by treatment of baicalein, which indicated inhibition of autophagic flux and lysosomal degradation (Figure 5E).

\section{Calcineurin and autophagy inhibition by PrPc deficiency protected against PrP (106-126)-induced neuroinflammation.}

One study showed that depleting PrPC blocks inhibits autophagic flux signals [28]. However, the relationship between autophagy and calcineurin signals in prion-mediated neuroinflammation is unknown. Thus, we investigated whether PrPC expression influences calcineurin-mediated autophagic flux signaling in $\operatorname{PrP}(106-126)$-treated neuronal cells. We cultured the mouse hippocampal neuron cell lines ZW 13-2 and Zpl 3-4, which were established from the hippocampus of ICR (Prnp $\left.{ }^{+/+}\right)$and Zürich I $\mathrm{Prnp}^{-/-}$mice, respectively. Real-time quantitative PCR analysis was then used to measure the mRNA levels of proinflammatory cytokines, TNF- $a$, and COX-2. We showed that prion peptide-induced TNF- $a$ and COX-2 mRNA under normal conditions, but PrPc deficiency attenuated prion peptide-induced TNF- $a$ and COX-2 mRNA in PrPc knockout Zpl 3-4 cells (Figure. 6A, B). Western blot assays showed that PrP(106126) treatment increase calcineurin and $p$-NF-KB levels in ZW 13-2 cells (Figure. 5C). Zpl 3-4 cells decreased the calcineurin and $p$-NF-KB protein levels compared to ZW-13-2 cells (Figure. 5D). Western blot assays showed that $\operatorname{PrP}(106-126)$ treatment inhibited autophagy flux in Zpl 3-4 cells (Figure 6E, F), and that PrPc expression was displayed in ZW 13-2 cells (Figure 6E). In contrast, no detectable PrPc was evident in Zpl 3-4 cells (Figure. 6F). These data indicate that PrPC expression plays a main role in calcineurin-mediated autophagic flux in $\operatorname{PrP}(106-126)$-induced inflammation. 


\section{Discussion}

The study was to investigate the role of melatonin in calcineurin inactivation and autophagy, and the regulation of prion protein-induced inflammation by melatonin in neuronal cells. The results suggest that the decrease in calcineurin and autophagy by melatonin and the consequent reduction in prion proteininduced neuroinflammation may be the key mechanisms underlying the neuroprotective effects of melatonin.

It has been reported that melatonin suppresses ER stress-mediated proapoptotic effects in bone mesenchymal stem cells [29]. Suwanjang $W$ et al suggested that the protective effect of melatonin is mediated via cytosolic calcium in DEX-induced neurotoxicity [30]. Melatonin prevents hydrogen peroxide in human neuroblastoma cells by attenuating calcineurin signaling pathways [31]. Consistent with these findings, our results also demonstrated that melatonin attenuates calcineurin activity. Together, these findings suggest that melatonin effectively inhibits prion protein-induced calcium and calcineurin activity.

Because melatonin has neuroprotective and antioxidant properties [32], It has been reported to exert a neuroprotective effect against ß-amyloid peptide [33], aging-related neurodegenerative disease [34], and human prion protein fragment 106-126 [35]. Because of its anti-inflammatory, antioxidant, and antiapoptotic properties, melatonin exerts neuroprotective actions against different neurological diseases. This study explored the neuroprotective role of melatonin against prion protein-induced neuroinflammation.

A relation of calcineurin and NF-KB has been previously reported [36, 37]. Calcineurin is an upstream regulator of NF-KB activation and calcineurin and NF-KB inhibition attenuated isoflurane-induced neuroinflammation and subsequent cognitive impairment [38]. Calcineurin functions as a critical signaling molecule during $\mathrm{T}$ helper cell activation, regulating $\mathrm{Bcl}-10$ phosphorylation and thereby NF-KB activation [39]. And Our unpublished data demonstrated that calcineurin regulates $\operatorname{PrP}(106-126)$-induced neuroinflammation by autophagy. The results of the present study suggest that melatonin inactivates calcineurin activation and NF-KB following autophagy.

We previously reported that melatonin-induced autophagy on $\operatorname{PrP}(106-126)$-mediated neurotoxicity and mitochondrial dysfunction [21]. However, effect of melatonin-reduced calcineurin activation and autophagy activation in $\operatorname{PrP}(106-126)$-induced neuroinflammation has yet to be reported. Our present study demonstrated that melatonin treatment protects against $\operatorname{PrP}(106-126)$-induced neuroinflammation by regulation of calcineurin and autophagy inactivation..

Melatonin significantly increased the level of autophagy in prion protein-induced apoptosis [21]. However, other studies report that melatonin attenuated autophagy $[40,41]$. In the present study, we showed that melatonin inhibited autophagy in neuronal cells. Autophagic defects are well documented in neurodegenerative diseases, and include accumulation of autophagosomes and undegraded autophagic vacuoles in neuronal cells [27]. 


\section{Conclusion}

In our future study, we will further explore the neuroprotective effects of melatonin, autophagy and the calcineurin pathway in mouse models to examine melatonin's potential therapeutic role in prion disease.

\section{Abbreviations}

Mel: melatonin; CNS: central nervous system; PrPc: cellular prion protein; PrPsc: scrapie-associated prion protein; LC3-I/II: microtubule-associated protein 1A/1B-light chain 3-I/II; CQ: chloroquine

\section{Declarations}

\section{Ethics approval and consent to participate}

Not applicable

\section{Consent for publication}

Not applicable

\section{Availability of data and materials}

The datasets used and/or analyzed during the current study are available from the corresponding author on reasonable request.

\section{Competing interests}

The authors declare that they have no competing interests

\section{Funding}

This study was supported by National Research Foundation of the Korea Grant (NRF) which was funded by Ministry of Education (2019R1A6A1A03033084). NRF did not play a role in the study design or analyses.

\section{Author information}

$\mathrm{JH}$ and $\mathrm{JM}$ analyzed and interpreted all the data. $\mathrm{JH}$ performed the experiments and was a major contributor in writing the manuscript. JS and SP contributed to study design and the writing of the manuscript. All authors read and approved the final manuscript.

\section{Acknowledgments}

Not applicable. 


\section{References}

1. Prusiner SB: Prion diseases and the BSE crisis.Science 1997, 278:245-251.

2. Prusiner SB: Molecular biology of prion diseases.Science 1991, 252:1515-1522.

3. Aguzzi A, Calella AM: Prions: protein aggregation and infectious diseases. Physiological reviews 2009, 89:1105-1152.

4. Minghetti L: Role of COX-2 in inflammatory and degenerative brain diseases.Subcell Biochem 2007, 42:127-141.

5. Bradford BM, Mabbott NA: Prion disease and the innate immune system. Viruses 2012, 4:3389-3419.

6. Forloni G, Angeretti N, Chiesa R, Monzani E, Salmona M, Bugiani O, Tagliavini F: Neurotoxicity of a prion protein fragment.Nature 1993, 362:543.

7. Forloni G, Angeretti N, Chiesa R, Monzani E, Salmona M, Bugiani O, Tagliavini F: Neurotoxicity of a prion protein fragment.Nature 1993, 362:543-546.

8. Selvaggini C, De Gioia L, Cantu L, Ghibaudi E, Diomede L, Passerini F, Forloni G, Bugiani O, Tagliavini F, Salmona M: Molecular characteristics of a protease-resistant, amyloidogenic and neurotoxic peptide homologous to residues 106-126 of the prion protein.Biochem Biophys Res Commun 1993, 194:1380-1386.

9. Hardeland R: Melatonin and the theories of aging: a critical appraisal of melatonin's role in antiaging mechanisms.J Pineal Res 2013, 55:325-356.

10. Mauriz JL, Collado PS, Veneroso C, Reiter RJ, Gonzalez-Gallego J: A review of the molecular aspects of melatonin's anti-inflammatory actions: recent insights and new perspectives.J Pineal Res 2013, 54:1-14.

11. Das A, McDowell M, Pava MJ, Smith JA, Reiter RJ, Woodward JJ, Varma AK, Ray SK, Banik NL: The inhibition of apoptosis by melatonin in VSC4.1 motoneurons exposed to oxidative stress, glutamate excitotoxicity, or TNF-alpha toxicity involves membrane melatonin receptors.J Pineal Res 2010, 48:157-169.

12. Corpas R, Grinan-Ferre C, Palomera-Avalos V, Porquet D, Garcia de Frutos P, Franciscato Cozzolino SM, Rodriguez-Farre E, Pallas M, Sanfeliu C, Cardoso BR: Melatonin induces mechanisms of brain resilience against neurodegeneration.J Pineal Res 2018, 65:e12515.

13. Crabtree GR: Generic signals and specific outcomes: signaling through $\mathrm{Ca} 2+$, calcineurin, and NFAT.Cell 1999, 96:611-614.

14. Agostinho P, Lopes JP, Velez Z, Oliveira CR: Overactivation of calcineurin induced by amyloid-beta and prion proteins. Neurochem Int 2008, 52:1226-1233.

15. Goto S, Matsukado Y, Mihara Y, Inoue N, Miyamoto E: The distribution of calcineurin in rat brain by light and electron microscopic immunohistochemistry and enzyme-immunoassay.Brain Res 1986, 397:161-172.

16. Goto S, Matsukado Y, Mihara Y, Inoue N, Miyamoto E: Calcineurin in human brain and its relation to extrapyramidal system. Immunohistochemical study on postmortem human brains.Acta 
Neuropathol 1986, 72:150-156.

17. Palkowitsch L, Marienfeld U, Brunner C, Eitelhuber A, Krappmann D, Marienfeld RB: The Ca2+dependent phosphatase calcineurin controls the formation of the Carma1-Bcl10-Malt1 complex during T cell receptor-induced NF-kappaB activation.J Biol Chem 2011, 286:7522-7534.

18. Klionsky DJ, Abdelmohsen $\mathrm{K}$, Abe A, Abedin MJ, Abeliovich $\mathrm{H}$, Acevedo Arozena A, Adachi $\mathrm{H}$, Adams $\mathrm{CM}$, Adams PD, Adeli K, et al: Guidelines for the use and interpretation of assays for monitoring autophagy (3rd edition).Autophagy 2016, 12:1-222.

19. Yorimitsu T, Klionsky DJ: Autophagy: molecular machinery for self-eating.Cell Death Differ 2005, 12 Suppl 2:1542-1552.

20. Levine B, Kroemer G: Biological Functions of Autophagy Genes: A Disease Perspective.Cell 2019, 176:11-42.

21. Jeong JK, Moon MH, Lee YJ, Seol JW, Park SY: Melatonin-induced autophagy protects against human prion protein-mediated neurotoxicity.J Pineal Res 2012, 53:138-146.

22. Moon JH, Park SY: Baicalein prevents human prion protein-induced neuronal cell death by regulating JNK activation.Int J Mol Med 2015, 35:439-445.

23. Corsaro A, Thellung S, Villa V, Principe DR, Paludi D, Arena S, Millo E, Schettini D, Damonte G, Aceto A, et al: Prion protein fragment 106-126 induces a p38 MAP kinase-dependent apoptosis in SH-SY5Y neuroblastoma cells independently from the amyloid fibril formation.Ann N Y Acad Sci 2003, 1010:610-622.

24. Moon JH, Jeong JK, Hong JM, Seol JW, Park SY: Inhibition of Autophagy by Captopril Attenuates Prion Peptide-Mediated Neuronal Apoptosis via AMPK Activation.Mol Neurobiol 2019, 56:4192-4202.

25. Hong JM, Moon JH, Park SY: Human prion protein-mediated calcineurin activation induces neuron cell death via AMPK and autophagy pathway.Int J Biochem Cell Bio/2020, 119:105680.

26. Lee JH, Moon JH, Kim SW, Jeong JK, Nazim UM, Lee YJ, Seol JW, Park SY: EGCG-mediated autophagy flux has a neuroprotection effect via a class III histone deacetylase in primary neuron cells.Oncotarget 2015, 6:9701-9717.

27. Moon JH, Park SY: Prion peptide-mediated calcium level alteration governs neuronal cell damage through AMPK-autophagy flux.Cell Commun Signal 2020, 18:109.

28. Moon JH, Lee JH, Nazim UM, Lee YJ, Seol JW, Eo SK, Lee JH, Park SY: Human prion protein-induced autophagy flux governs neuron cell damage in primary neuron cells. Oncotarget 2016, 7:2998930002.

29. Fan C, Feng J, Tang C, Zhang Z, Feng Y, Duan W, Zhai M, Yan Z, Zhu L, Feng L, et al: Melatonin suppresses ER stress-dependent proapoptotic effects via AMPK in bone mesenchymal stem cells during mitochondrial oxidative damage.Stem Cell Res Ther 2020, 11:442.

30. Suwanjang W, Abramov AY, Charngkaew K, Govitrapong P, Chetsawang B: Melatonin prevents cytosolic calcium overload, mitochondrial damage and cell death due to toxically high doses of dexamethasone-induced oxidative stress in human neuroblastoma SH-SY5Y cells. Neurochem Int 2016, 97:34-41. 
31. Premratanachai A, Suwanjang W, Govitrapong P, Chetsawang J, Chetsawang B: Melatonin prevents calcineurin-activated the nuclear translocation of nuclear factor of activated T-cells in human neuroblastoma SH-SY5Y cells undergoing hydrogen peroxide-induced cell death.J Chem Neuroanat 2020, 106:101793.

32. Watson N, Diamandis T, Gonzales-Portillo C, Reyes S, Borlongan CV: Melatonin as an Antioxidant for Stroke Neuroprotection.Cell Transplant 2016, 25:883-891.

33. Ansari Dezfouli M, Zahmatkesh M, Farahmandfar M, Khodagholi F: Melatonin protective effect against amyloid beta-induced neurotoxicity mediated by mitochondrial biogenesis; involvement of hippocampal Sirtuin-1 signaling pathway.Physiol Behav 2019, 204:65-75.

34. Luo F, Sandhu AF, Rungratanawanich W, Williams GE, Akbar M, Zhou S, Song BJ, Wang X: Melatonin and Autophagy in Aging-Related Neurodegenerative Diseases.Int J Mol Sci 2020, 21.

35. Jeong JK, Lee JH, Moon JH, Lee YJ, Park SY: Melatonin-mediated beta-catenin activation protects neuron cells against prion protein-induced neurotoxicity.J Pineal Res 2014, 57:427-434.

36. de la Fuente V, Federman N, Fustinana MS, Zalcman G, Romano A: Calcineurin phosphatase as a negative regulator of fear memory in hippocampus: control on nuclear factor-kappaB signaling in consolidation and reconsolidation. Hippocampus 2014, 24:1549-1561.

37. Lim D, lyer A, Ronco V, Grolla AA, Canonico PL, Aronica E, Genazzani AA: Amyloid beta deregulates astroglial mGluR5-mediated calcium signaling via calcineurin and Nf-kB.Glia 2013, 61:1134-1145.

38. Li Z, Ni C, Xia C, Jaw J, Wang Y, Cao Y, Xu M, Guo X: Calcineurin/nuclear factor-kappaB signaling mediates isoflurane-induced hippocampal neuroinflammation and subsequent cognitive impairment in aged rats.Mol Med Rep 2017, 15:201-209.

39. Frischbutter S, Gabriel C, Bendfeldt H, Radbruch A, Baumgrass R: Dephosphorylation of Bcl-10 by calcineurin is essential for canonical NF-kappaB activation in Th cells. Eur $\mathrm{J}$ Immunol 2011, 41:23492357.

40. Feng Y, Xu F, Wang SM, Wu SX, Zhang XH, Gao YX, Li YL, Zhong DB, Yin JZ, Feng YM: Melatonin attenuates nicotine-induced autophagy and neurological changes by decreasing the production of reactive oxygen species.Int J Neurosci 2020, 130:391-397.

41. Ali T, Rahman SU, Hao Q, Li W, Liu Z, Ali Shah F, Murtaza I, Zhang Z, Yang X, Liu G, Li S: Melatonin prevents neuroinflammation and relieves depression by attenuating autophagy impairment through FOXO3a regulation.J Pineal Res 2020, 69:e12667.

\section{Figures}



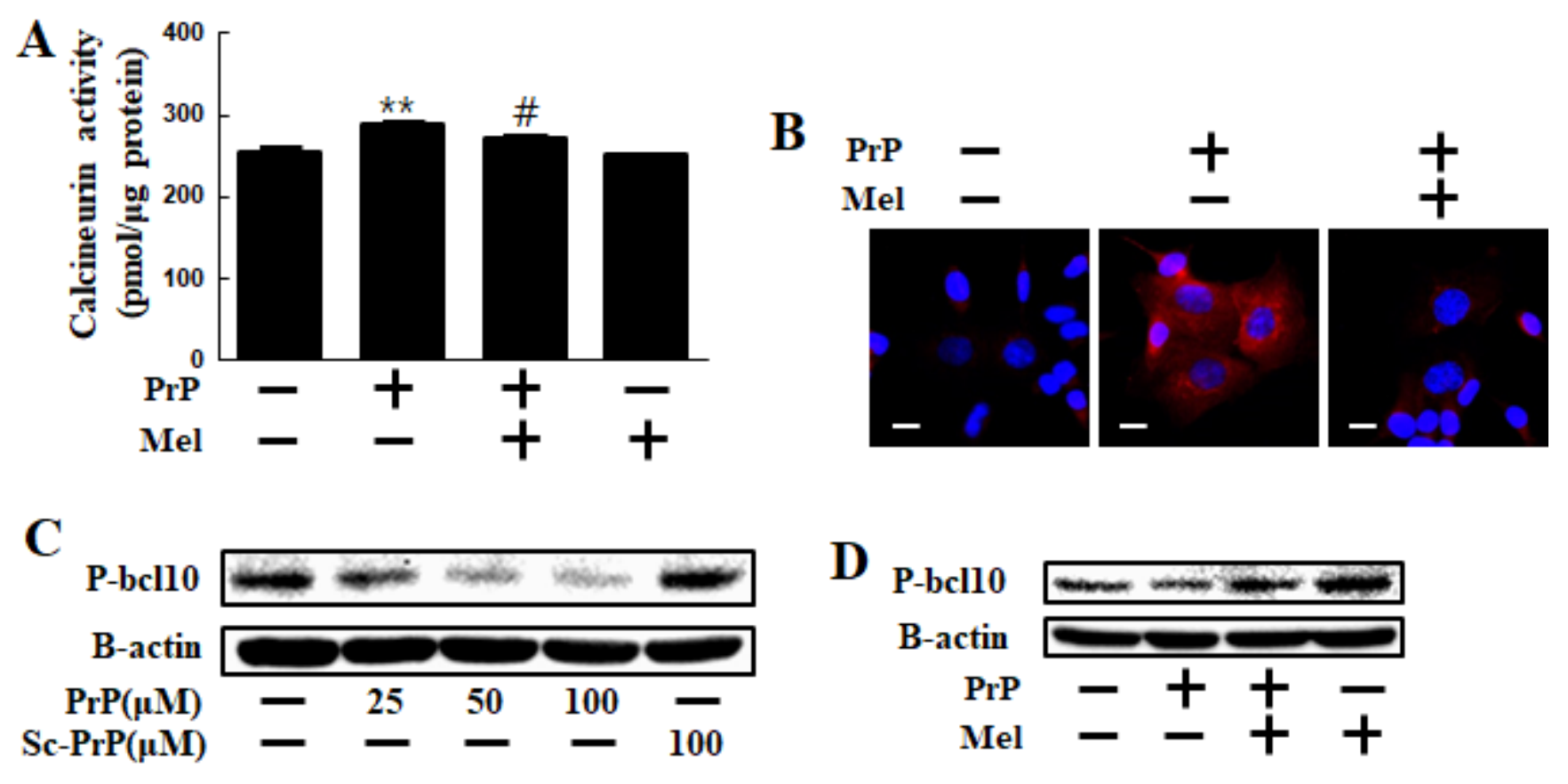

Figure 1

Effect of melatonin against PrP (106-126)-induced inflammation in neuronal cells. (A) SK-N-SH cells were treated with melatonin for $1 \mathrm{~h}$ and then exposed to $100 \mu \mathrm{M}$ of $\operatorname{PrP}(106-126)$ for $3 \mathrm{~h}$. Total RNA was extracted to quantify the mRNA expression of TNF alpha. (B) Total RNA was extracted to quantify the mRNA expression of COX-2. (C) SK-N-SH cells were treated with melatonin for $1 \mathrm{~h}$ and then exposed to $100 \mu \mathrm{M}$ of $\operatorname{PrP}$ (106-126) for $12 \mathrm{~h}$. The treated cells were assessed for $\mathrm{p}-\mathrm{NF}-\mathrm{kB}$ by Western blot analysis.

(D) Immunocytochemistry for the p-NF-kB was analyzed from neuroblastoma cells. The bar graph shows the mean \pm standard error of the mean $(n=3)$. Magnification 200x, scale bar $=50 \mu \mathrm{m} .{ }^{*} p<0.001$, significant differences when comparing the control group with each treatment group. PrP, Prion peptide $(106-126)$ 

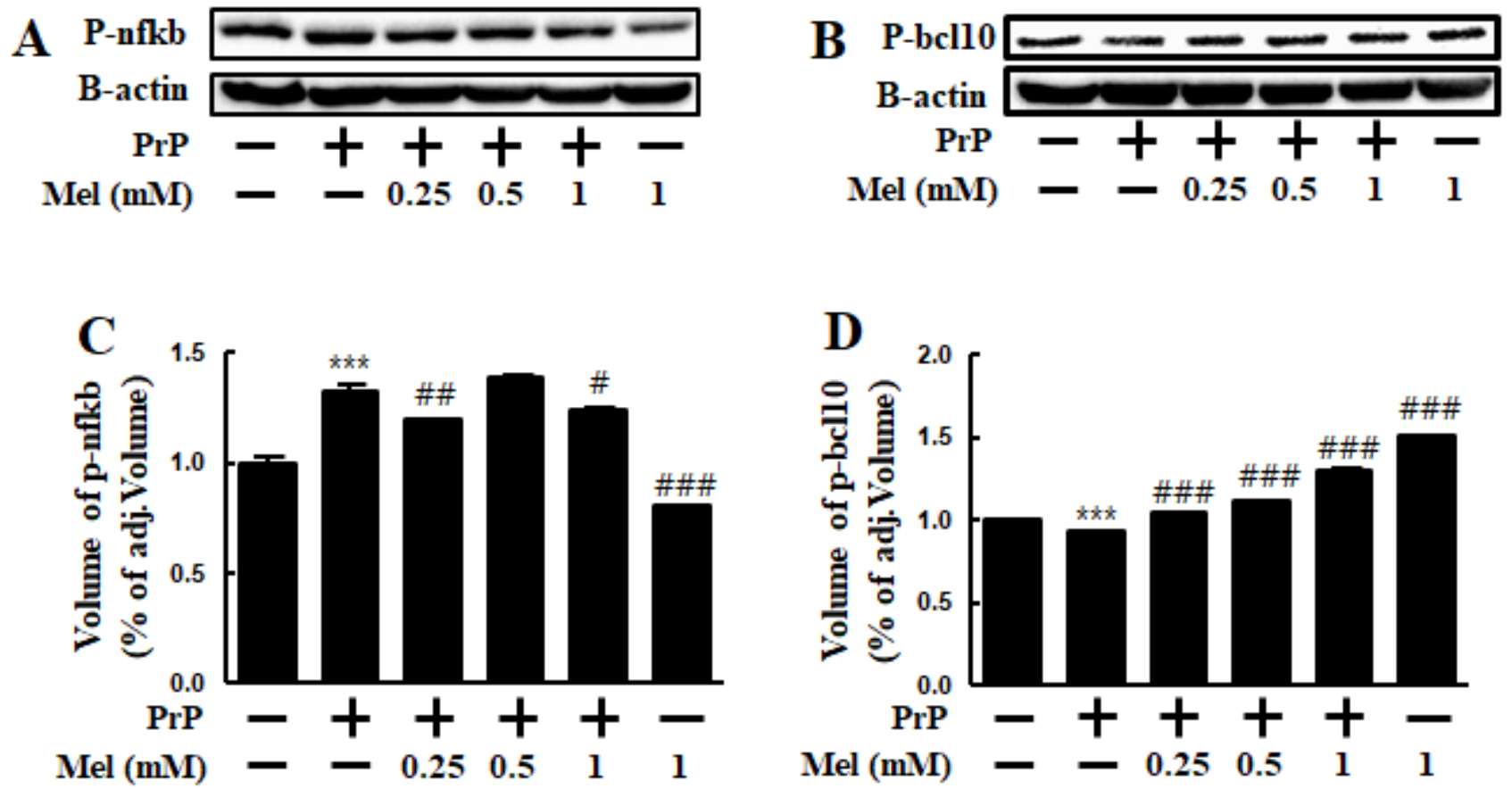

Figure 2

Melatonin attenuates PrP (106-126)-induced p-NF-kB-mediated inflammation. (A) SK-N-SH cells were treated with melatonin for $1 \mathrm{~h}$ and then exposed to $100 \mu \mathrm{M}$ of $\operatorname{PrP}(106-126)$ for $12 \mathrm{~h}$. The treated cells were assessed for p-NF-kB by Western blot analysis. (B) The treated cells were assessed for $\mathrm{p}-\mathrm{bcl} 10$ by Western blot analysis. (C) Bar graph indicated that volume of p-NF-kB expression levels. (D) Bar graph indicated that volume of $\mathrm{p}$-bcl10 expression levels. The bar graph shows the mean \pm standard error of the mean $(n=3)$. Magnification 200x, scale bar $=50 \mu \mathrm{m}$. *** $p<0.001$, \#\# $p<0.01$, \#\#\#p<0.001; significant differences when comparing the control group with each treatment group. PrP, Prion peptide (106-126) 

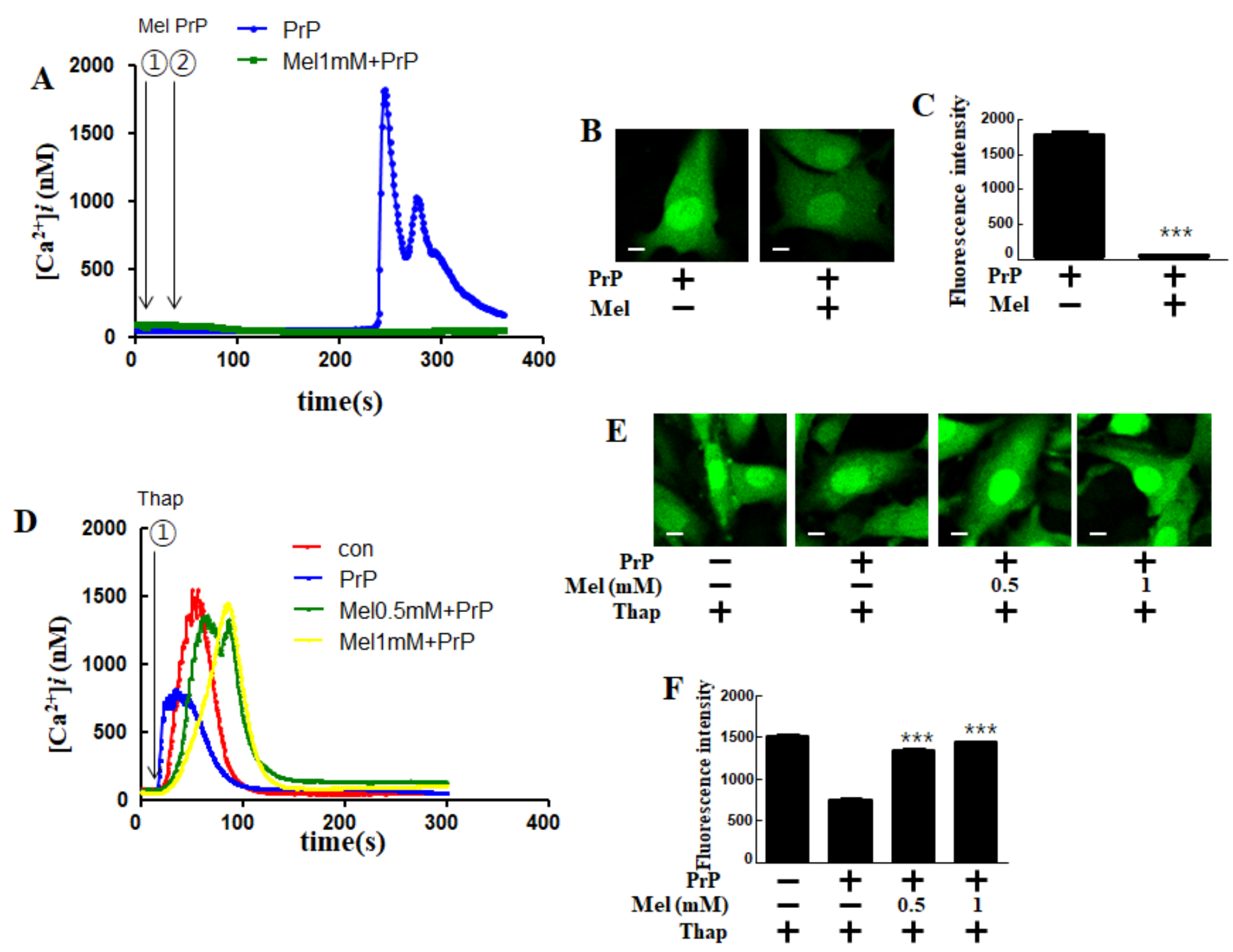

Figure 3

Melatonin inhibits PrP (106-126)-induced neuroinflammation through calcium. (A) SK-N-SH cells were loaded with fluo-4 AM and the changes in $\mathrm{Ca} 2+$ levels were measured using confocal microscope. SK-N$\mathrm{SH}$ cells were treated with melatonin and calcineurin inhibitor for $1 \mathrm{~h}$ and then exposed to $100 \mu \mathrm{M}$ of $\operatorname{PrP}$ (106-126) for $12 \mathrm{~h}$. The time point of $1 \mu \mathrm{M}$ of thapsigargin addition is indicated by the $\nabla$ arrow. Data are mean \pm SEM of [Ca2+]i at $60 \mathrm{~s}$ from three independent experiments. (B) Bar graph indicating the average peak value of intracellular calcium levels. (C) The green fluorescence image indicates peak value of calcium measurement using confocal microscopy. (D) SK-N-SH cells were loaded with fluo-4 AM and the changes in $\mathrm{Ca} 2+$ levels were measured using confocal microscope. The time point of $40 \mu \mathrm{M}$ of melatonin addition is indicated by the $\otimes$ arrow and $100 \mu \mathrm{M}$ of $\operatorname{PrP}(106-126)$ addition is indicated by the $\otimes$ arrow. Data are mean \pm SEM of [Ca2+]i at $60 \mathrm{~s}$ from three independent experiments. (E) Bar graph indicating the average peak value of intracellular calcium levels. (F) The green fluorescence image indicates peak value of calcium measurement using confocal microscopy. The bar graph shows the mean \pm standard error of the mean from three independent experiments. Magnification 200x, scale bar $=50 \mu \mathrm{m}$. ${ }^{\star \star \star} p<0.001, \# p<$ 
0.05; significant differences when compared with the control group and each treatment group. PrP, Prion peptide (106-126)
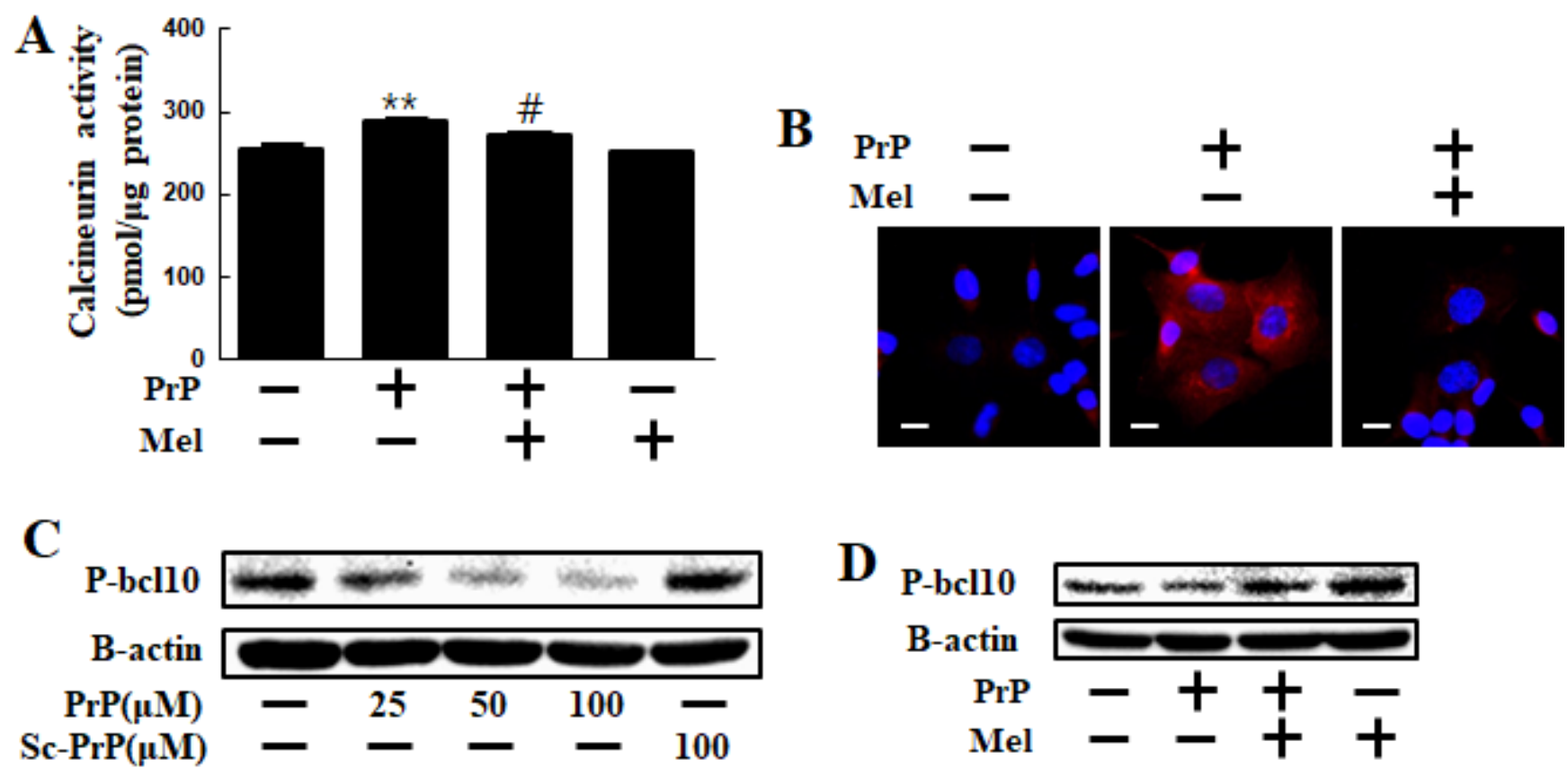

\section{Figure 4}

Melatonin inhibits PrP (106-126)-induced neuroinflammation via calcineurin inhibition. (A) SK-N-SH cells were treated with melatonin for $1 \mathrm{~h}$ and then exposed to $100 \mu \mathrm{M}$ of PrP (106-126) for $12 \mathrm{~h}$. The treated cells were assessed for calcineurin activity. (B) Immunocytochemistry for the calcineurin was analyzed from neuroblastoma cells. (C) SK-N-SH cells were treated with 25, 50, and $100 \mu \mathrm{M}$ of PrP (106-126) and $100 \mu \mathrm{M}$ Sc-PrP (106-126) for $12 \mathrm{~h}$. The treated cells were assessed for p-bcl10 by Western blot analysis. (D) SK-N-SH cells were treated with melatonin for $1 \mathrm{~h}$ and then exposed to $100 \mu \mathrm{M}$ of PrP (106-126) for $12 \mathrm{~h}$. The treated cells were assessed for p-bcl10 by Western blot analysis. The bar graph shows the mean \pm standard error of the mean $(n=3)$. Magnification $200 x$, scale bar $=50 \mu \mathrm{m}$. *** $p<0.001, \# \# p<$ 0.01 , \#\#\# $\mathrm{p}<0.001$; significant differences when comparing the control group with each treatment group. PrP, Prion peptide (106-126) 

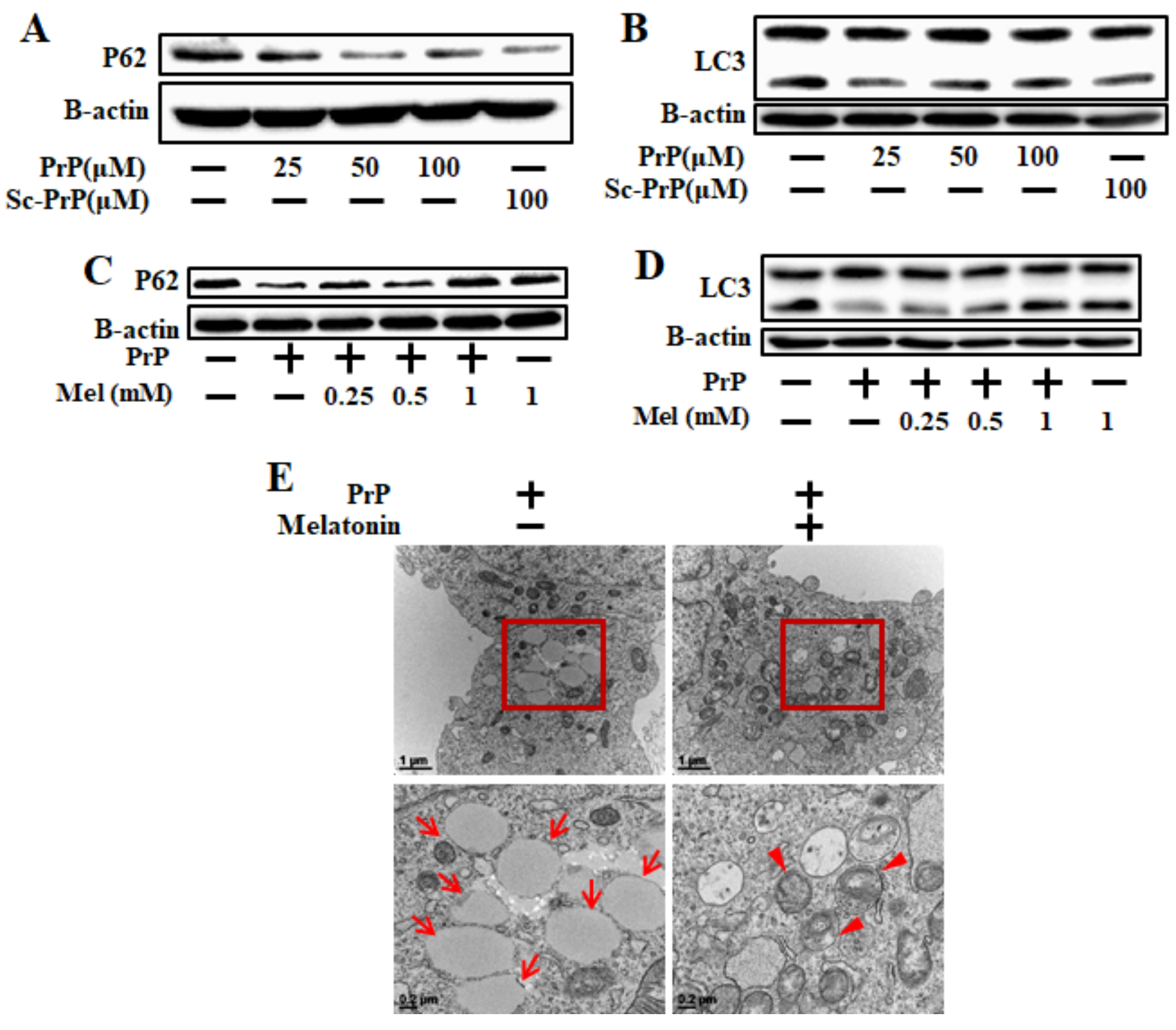

Figure 5

Melatonin inhibits PrP (106-126)-induced autophagy. (A) SK-N-SH cells were treated with 25, 50, and 100 $\mu \mathrm{M}$ of PrP (106-126) and $100 \mu \mathrm{M}$ Sc-PrP (106-126) for $12 \mathrm{~h}$. The treated cells were assessed for P62 by Western blot analysis. (B) The treated cells were assessed for LC3 by Western blot analysis. (C) SK-N-SH cells were treated with $0.25,0.5$ and $1 \mathrm{mM}$ of melatonin for $1 \mathrm{~h}$ and then exposed to $100 \mu \mathrm{M}$ of PrP (106126) for $12 \mathrm{~h}$ The treated cells were assessed for $\mathrm{P} 62$ by Western blot analysis. (D) The treated cells were assessed for LC3 by Western blot analysis. (E) SK-N-SH cells were treated with melatonin for $1 \mathrm{~h}$ and then exposed to $100 \mu \mathrm{M}$ of PrP (106-126) for $12 \mathrm{~h}$ and analyzed by TEM. Arrows indicate autophagosomes and arrowheads indicate lysosomes and mitochondria. Magnification 200x, scale bar =50 $\mu \mathrm{m}$. 

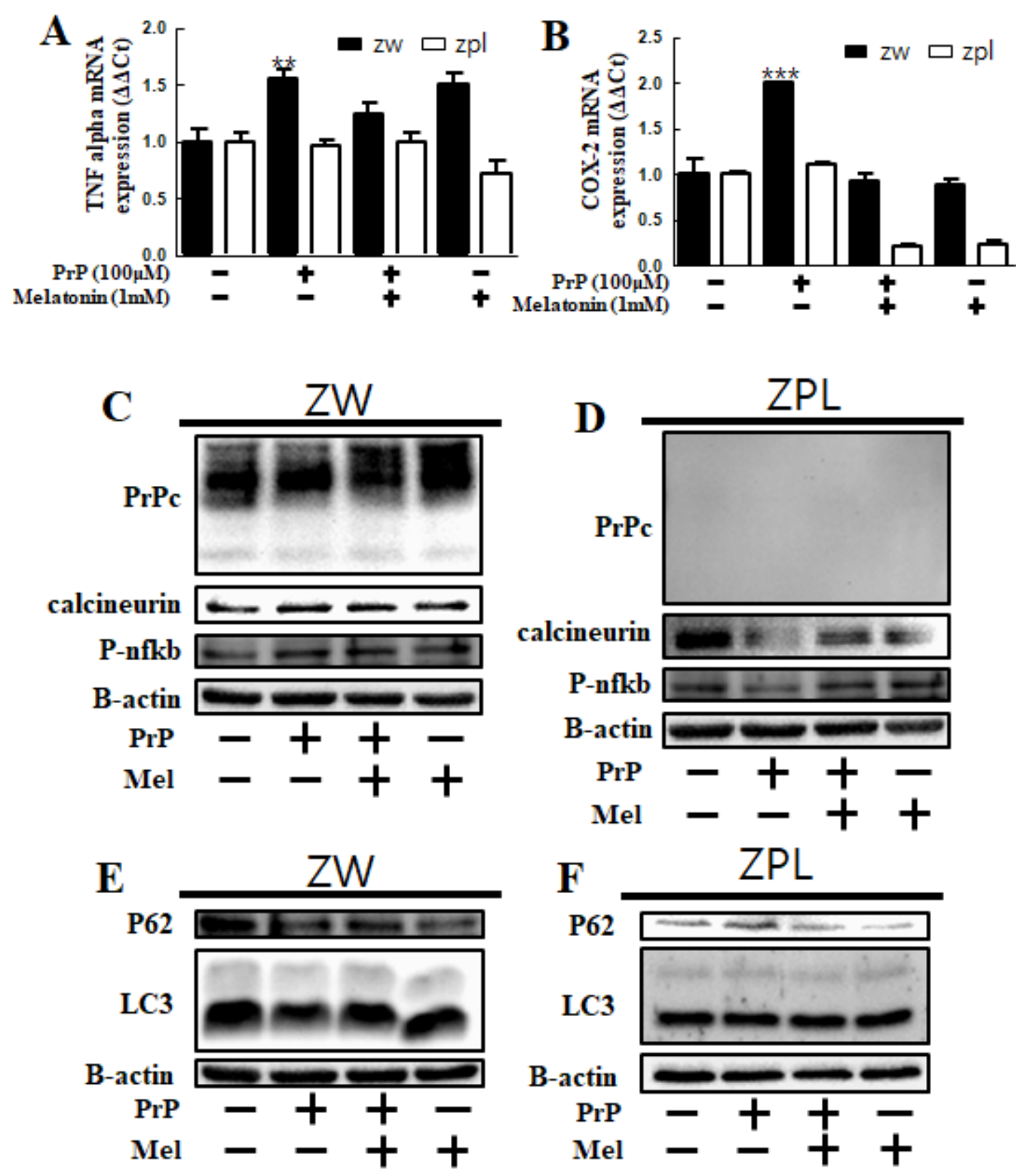

Figure 6

Melatonin inhibited $\operatorname{PrP}(106-126)$-induced neuroinflammation in Zw cell. (A) Zw and Zpl cells were treated with melatonin for $1 \mathrm{~h}$ and then exposed to $100 \mu \mathrm{M}$ of $\operatorname{PrP}(106-126)$ for $3 \mathrm{~h}$. Total RNA was extracted to quantify the mRNA expression of TNF alpha. (B) Total RNA was extracted to quantify the mRNA expression of COX-2. (C) SK-N-SH cells were treated with melatonin for $1 \mathrm{~h}$ and then exposed to $100 \mu \mathrm{M}$ of PrP (106-126) for $12 \mathrm{~h}$. The treated cells were assessed for PrPc, calcineurin and p-NF-kB by Western blot analysis in ZW cells. (D) The treated cells were assessed for PrPc, calcineurin and p-NF-kB by Western blot analysis in Zpl cells. (E) SK-N-SH cells were treated with melatonin for $1 \mathrm{~h}$ and then exposed to $100 \mu \mathrm{M}$ of PrP (106-126) for $12 \mathrm{~h}$. The treated cells were assessed for P62 and LC3 by Western blot analysis in ZW cells. (F) The treated cells were assessed for P62 and LC3 by Western blot analysis in Zpl cells. The bar graph shows the mean \pm standard error of the mean $(n=3)$. ${ }^{\star} p<0.001$, 
significant differences when comparing the control group with each treatment group. PrP, Prion peptide $(106-126)$ 